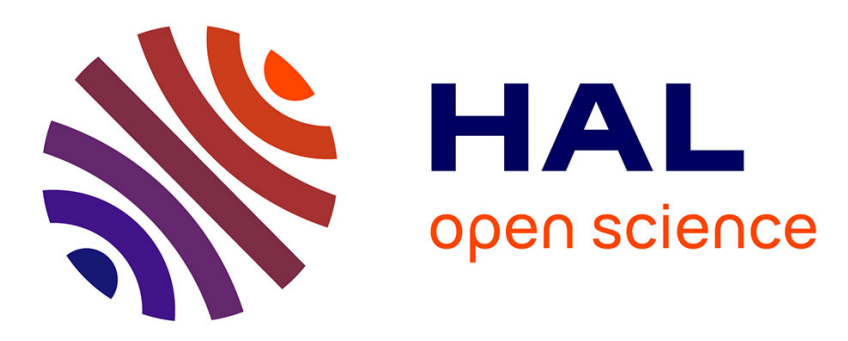

\title{
Mobile Robots Cooperation with Biased Exteroceptive Measurements
}

Khaoula Lassoued, Oana Stanoi, Philippe Bonnifait, Isabelle Fantoni

\section{To cite this version:}

Khaoula Lassoued, Oana Stanoi, Philippe Bonnifait, Isabelle Fantoni. Mobile Robots Cooperation with Biased Exteroceptive Measurements. 13th International Conference on Control, Automation, Robotics and Vision (ICARCV 2014), Dec 2014, Marina Bay Sands, Singapore. pp.1838-1840. hal01098024

\section{HAL Id: hal-01098024 \\ https://hal.science/hal-01098024}

Submitted on 22 Dec 2014

HAL is a multi-disciplinary open access archive for the deposit and dissemination of scientific research documents, whether they are published or not. The documents may come from teaching and research institutions in France or abroad, or from public or private research centers.
L'archive ouverte pluridisciplinaire HAL, est destinée au dépôt et à la diffusion de documents scientifiques de niveau recherche, publiés ou non, émanant des établissements d'enseignement et de recherche français ou étrangers, des laboratoires publics ou privés. 


\title{
Mobile Robots Cooperation with Biased Exteroceptive Measurements
}

\author{
Khaoula Lassoued, Oana Stanoi, Philippe Bonnifait and Isabelle Fantoni \\ Université de Technologie de Compiègne, \\ UMR CNRS 7253 Heudiasyc, \\ Compiègne, France \\ Email: \{khaoula.lassoued, oana.stanoi, philippe.bonnifait, isabelle.fantoni $\} @ u t c . f r$
}

\begin{abstract}
When mobile robots need to cooperate, mutual localization is a key issue. The objective is to enable cooperative localization capabilities, such that each robot determines the partners positions in a common frame with reliable confidence estimates. Exteroceptive sensors can measure distances to known beacons in order to provide absolute information. It often exists biases that affect these measurements because of particular environment conditions or because of an inaccurate knowledge of the beacons positions. In this work, each robot is also equipped with proprioceptive sensors, but no sensor can measure the interdistance between the robots. The method that we consider is fully distributed between the robots, which share positions and biases estimates. In order to handle the data incest problem, we use constraint propagation techniques on intervals. The distributed cooperative localization method gives sets that always contain the true positions of the robots without any over-convergence. Simulation results show that the so-called method improves localization performance compared to standalone methods.
\end{abstract}

\section{INTRODUCTION}

Mobile robot localization with reliable confidence indicators is vital to enable various cooperative robot applications. This becomes particularly important when it deals with heterogeneous robots [1]. Fusion between exteroceptive sensor data from Global Positioning System (GPS) or cameras [2] and proprioceptive sensor data from wheel encoders or gyroscopes attached on mobile robots [3] enables to get high frequency absolute pose estimation. In order to improve absolute or relative position of robots, different approaches have been developed. The well known approaches are probabilistic methods relying on Extended Kalman Filtering (EKF) [4], Particle Filtering [5] or Least Square Estimation [6]. These methods do not provide guaranted position estimates. Moreover, they rely on the hypothesis of error independence and have to address the problem of data-incest. Aufrere et al. [7] solved this problem by handling private copies of the knowledge of robot group to address this over-convergence issue.

Advanced applications depend more on guaranteed position to enable a safe rendez-vous or collision free swarm navigation for example. An interesting solution is to use a constraint-based approach giving sets that always contain the true positions of robots without any over-convergence with the only assumption that model and measurements errors are bounded. Meizel et al.[8] developed methods based on bounded-error observers with set inversion. A high precision can be reached but it is not suitable to be applied in real time because of the high computational time arising from bisections. Other set-membership methods based on Constraint Propagation (CP) can deal with real-time applications when there is a high redundancy of measurements [9]. These methods have been applied to single robots but little in the context of cooperative localization. For instance, Drevelle et al. [10] exploited a group of Autonomous Underwater Vehicles (AUV) to characterize a large explored space and to monitor the area. In this application, they used range sensors to measure the inter-distance between robots. Kyoung-Hwan and Jihong have also studied a cooperative localization method for multiple ground robots based on CP techniques [11]. In their work, they supposed that each robot can communicate with the others and that it can share its position data but they didn't address the problem of the loss of communication, which likely occurs in practice. They also didn't take in consideration the biases on the measurements.

In this paper, we propose a new methodology for cooperative localization based on $\mathrm{CP}$ that guarantees that the true location of every robot is inside a confidence domain even if unknown measurement biases exist. Only two robots are taken in consideration in this paper for simplification, but the localization method can be extended to a heterogeneous system with $N$ robots such as ground and aerial robots. Robot positions can be estimated by using proprioceptive sensors and by using distance measurements given by fixed beacons but no sensor measures the inter-distance between the robots. We consider an environment where beacons with known location are far away from the evolution area of robots. The robots communicate in order to share positions and biases estimates. Two scenarios have been simulated. In the first, each robot has to localize itself in a standalone mode, and the second one concerns cooperative localization with full communication [11].

In section 2, the problem formulation is defined. In section 3 , the key steps of the proposed Distributed Cooperative Localization (DCL) algorithm based on CP techniques are detailed. In section 4, simulation results of two scenarios are shown and compared. These simulated results highlight the advantages of our approach. Finally, section 5 concludes the paper. 


\section{PROBLEM FORMULATION}

Let consider two robots equipped with exteroceptive sensors. We focus here on the use of range sensors.

\section{A. Exteroceptive measurements}

Range sensors are distance measurement devices widely used in robot navigation. They are often based on the principle of time-of-flight (GPS being a particular case because only pseudoranges can be measured). In our case, the sensors measure the distances to known active beacons via radio communication.

Let denote ${ }^{i} \rho^{j}$ a measured distance made by robot $i$ located at coordinates $\left(x_{i}, y_{i}\right)$ to an identified beacon $j(j=1 \ldots m)$ the position of which being known in the working frame. ${ }^{i} \rho^{j}$ is linked to the robot position via the observation model ${ }^{i} g^{j}$ :

$$
{ }^{i} \rho^{j}={ }^{i} g^{j}\left(x_{i}, y_{i}\right)
$$

The sensor provides measurements affected by noise and the coordinates of beacons are often known with some error. We consider a scenario where the beacons are far away from the evolution area. Under this hypothesis, the position errors of the beacons act as bias on the measurements. After linearization, the bias can be considered as an additive error, so the observation model for the robot $i$ becomes :

$$
{ }^{i} \rho^{j}={ }^{i} g^{j}\left(x_{i}, y_{i}\right)+b^{j}+{ }^{i} \beta^{j}
$$

where $b^{j}$ is the bias of beacon $j$, and ${ }^{i} \beta^{j}$ is the measurement error.

\section{B. Scenarios Description}

Standalone localization means that each robot estimates its position using its exteroceptive sensors data collected from the fixed beacons located in the evolution area. The cooperative localization of both robots means that each robot estimates its position with respect to beacons and also estimates the partner position in the working frame. The main difference with the standalone localization is that the position estimates can be improved thanks to the information exchange (position and biases estimates done by the partner). Figure (II.1) illustrates the scenario of the cooperation with shared information. The relative distance between the robots is denoted $\Delta$.

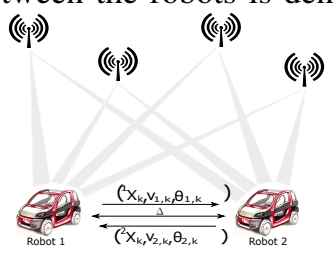

Figure II.1. Illustration of cooperative localization (in practice the distances with beacons are much higher than the distances between the robots)

Let ${ }^{i} X$ be the knowledge of the state vector of the system in robot $i, i=\{1,2\}$ in our case. $\left(x_{1, k}, y_{1, k}\right)$ and $\left(x_{2, k}, y_{2, k}\right)$ are the positions of robots 1 and 2 at each instant $k$ and ${ }^{i} b^{1} \ldots$ ${ }^{i} b^{m}$ are the biases estimates on the available exteroceptive measurements:

$$
{ }^{i} X_{k}=\left[\begin{array}{c}
{ }^{i} x_{1, k} \\
{ }^{i} y_{1, k} \\
{ }^{i} x_{2, k} \\
{ }^{i} y_{2, k} \\
{ }^{i} b^{1} \\
\vdots \\
{ }^{i} b^{m}
\end{array}\right]
$$

As long as a communication has been established (i.e. a robot can receive the information broadcast by the other), each robot can estimate the state of the system. But, if communication is lost, each robot updates its own state with its measurement and predicts the location of the other in order to continue to perform anyway the cooperative task.

As the exteroceptive measurements are biased, cooperative localization is very useful to correct those biases in a similar way to differential GPS done between a fixed base of known position and mobile rovers.

\section{Information exchange between robots}

We suppose that the robots have synchronized clocks working in the same time frame. For instance, in our case the synchronization can be done by Network Time Protocol (NTP), or by GPS. In our problem, accuracy of synchronization is not crucial.

Periodically, every robot broadcasts the following timestamped information:

- Its last position in the working frame,

- Its last estimate of the bias,

- The current velocity vector,

- The estimated position of the partner.

We use a set membership framework that is able to deal with public information and so it is not necessary to manipulate private copies of some information, as done in [1] and [7]. Every information is handled as an interval or a box. The estimation method is based on CP.

\section{COOPERATIVE LOCALIZATION ALGORITHM USING CONSTRAINT PROPAGATION TECHNIQUE}

\section{A. Cooperation algorithm}

The proposed architecture consists of a predictor/estimator mechanism that is illustrated in Figure (III.1). It is considered as a two-dimensional localization problem, with the $\left(x_{i}, y_{i}\right)$ coordinates and the bias ${ }^{i} b^{j}$ to be estimated. At any instant $k$, a position prediction uses the evolution model using the velocity and heading measured by proprioceptive sensors, and the previous position. The position estimation stage updates the predicted position with the new exteroceptive measurements to give the global position and bias estimation. 


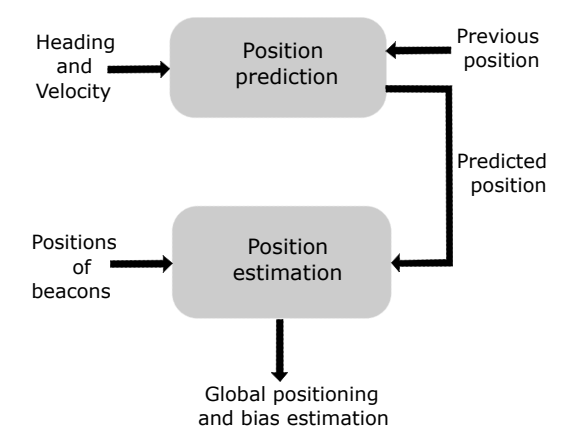

Figure III.1. Localization architecture for one robot

Proprioceptive measurements are made with a compas and wheel encoders. Hence, velocity and orientation information are obtained, which constitutes the input vector ${ }^{i} U_{k}$ applied on each robot. Considering the cooperative localization objective, ${ }^{i} U_{k}$ contains $v_{i, k}$ and $\theta_{i, k}$ measured by its own sensors and also the estimated $v_{i, k}$ and $\theta_{i, k}$ coming from the partner.

$$
{ }^{i} U_{k}=\left[\begin{array}{c}
{ }^{i} v_{1, k} \\
{ }^{i} \theta_{1, k} \\
{ }^{i} v_{2, k} \\
{ }^{i} \theta_{2, k}
\end{array}\right]
$$

Exteroceptive sensors provide partial information about the position of each robot considering the known locations of the beacons, which constitutes the observation model ${ }^{i} Y$. It contains the distances measured from each robots to the beacons.

$$
\begin{gathered}
{ }^{i} Y=\left[\begin{array}{c}
i \rho^{1} \\
i \\
\rho^{2} \\
\vdots \\
{ }^{i} \rho^{m}
\end{array}\right] \\
{ }^{1} \rho^{j}=\sqrt{\left(x_{1}-x_{j}\right)^{2}+\left(y_{1}-y_{j}\right)^{2}}+b^{j}+{ }^{1} \beta^{j} \\
{ }^{2} \rho^{j}=\sqrt{\left(x_{2}-x_{j}\right)^{2}+\left(y_{2}-y_{j}\right)^{2}}+b^{j}+{ }^{2} \beta^{j}
\end{gathered}
$$

where $j$ is the index of beacons.

When each robot has localized itself, it shares its estimated data with the other. The position of Robot 1 and the estimated position and uncertainty of Robot 2 by Robot 1 can be merged to obtain the cooperative localization. After the exchange of information has been made, each robot can predict the partner position using its last received information, considering the state vector ${ }^{i} X_{k}$ of each robot at time step $k$.

The robot location is described by two parameters $\left(x_{i}, y_{i}\right)$. Each robot predicts its position based on the evolution model described in Eq (III.5) ( $T_{e}$ is the sampling period) with the bias model in Eq (III.6):

$$
\left\{\begin{array}{c}
x_{i, k}=x_{i, k-1}+T_{e} v_{i, k-1} \cos \theta_{i, k-1} \\
y_{i, k}=y_{i, k-1}+T_{e} v_{i, k-1} \sin \theta_{i, k-1}
\end{array}\right.
$$

$$
b_{k}^{j}=b_{k-1}^{j}
$$

Finally, the cooperative localization algorithm for single robot is given below:

\begin{tabular}{l}
\hline Algorithm 1 Information processing in Robot $i$ \\
The robot \\
1) Makes its proprioceptive measurements \\
2) Predicts its position \\
3) Predicts the biases \\
4) Makes its exteroceptive measurements \\
5) Updates its own position \\
6) Predicts position of the partner using its last known \\
estimate of the velocity \\
7) Broadcasts its information to the partner \\
8) Listens if it has received information from the partner. \\
If positive, then the cooperation it made available: \\
a) Updates the prediction of the partner position using \\
the received partner position \\
b) Updates the biases
\end{tabular}

All these processes are executed by each robot and the cooperative localization is obtained by contraction of their state estimations. In this implementation of the method, the communication delays have to be smaller than the processing period. If not, stage 8 of the algorithm can be extended with data buffers to do the update in the past and propagate it up to the current time.

\section{B. Information fusion using Constraint Propagation}

In this paper, we extend the classical stand-alone estimation method based on $\mathrm{CP}$ to develop a cooperative data fusion algorithm.

In this problem the CSP $\mathcal{H}$ for each robot $i$ is :

$$
\mathcal{H}:\left(F(\mathbf{x})=\left[{ }^{i} g^{j}(\mathbf{x})\right]-\left[{ }^{i} \rho^{j}\right]=\mathbf{0}\right)
$$

Where $j=1 \ldots m$ represents the index of the beacon and $F$ is a vector function of $j$ constraints. The objective in our case is to contract $\mathcal{H}$ to eliminate the inconsistent part of the box state vector $\left[{ }^{i} X_{k}\right]$ with respect to the measurements. It is important to keep in mind that as long as the measurements are consistent with the chosen bounded error model, the solution set will include the true robot location.

A brief description of CSP and contractors is respectively given in section (III-B1) and (III-B2) :

1) Constraint Satisfaction Problem: Consider $n$ variables $x_{i} \in \mathbb{R}, i \in\{1, \ldots, n\}$, linked by $m$ relations (or constraints) of the form:

$$
f_{j}\left(x_{1}, \ldots, x_{n}\right)=0, j=1 \ldots m
$$

Each variable $x_{i}$ is known to belong to a domain $\mathrm{X}_{i}$. For simplicity, these domains will be intervals, denoted by $\left[x_{i}\right]$. Define the vector $\mathbf{x}=\left(x_{1}, \ldots, x_{n}\right)^{T}$ and its domain as a Cartesian product of $n$ intervals $\left([\mathbf{x}]=\otimes\left[x_{i}\right]\right)$. As figure (III.2), each interval is written in boundary as : $[x]=\{x \mid \underline{x} \leq x \leq \bar{x}\}$. 


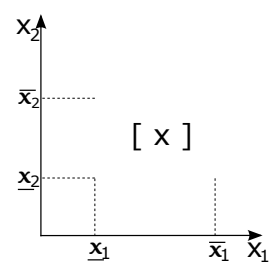

Figure III.2. A box

Knowing that $F$ is the function whose coordinate functions are the $f_{j}$, Eq (III.8) can then be written in vector form $F(\mathbf{x})=\mathbf{0}$. Searching the minimal box of $[\mathbf{x}]$ satisfying all the constraints $f_{j}$ corresponds to a CSP denoted by $\mathcal{H}$ :

$$
\mathcal{H}:(F(\mathbf{x})=\mathbf{0} \mid \mathbf{x} \in[\mathbf{x}])
$$

The solution set of $\mathcal{H}$ is defined as:

$$
S=\{\mathbf{x} \in[\mathbf{x}] \mid F(\mathbf{x})=\mathbf{0}\}
$$

For more details about the CSP, please refer to [12].

2) Contractors: Contracting $\mathcal{H}$ means replacing $[\mathrm{x}]$ by a smaller domain $\left[\mathrm{x}^{\prime}\right]$ such that the solution set remains unchanged, and $S \subset\left[\mathbf{x}^{\prime}\right] \subset[\mathbf{x}]$. A contractor for $\mathcal{H}$ is defined as an operator used to contract $\mathcal{H}$.

There are different kinds of contractors [12] developed in order to reduce the pessimism of a returned box. In this paper, a Forward-Backward contractor based on primitive constraint [13] is adopted, because of the great redundancy of data and equations, and because of the independence of the method to non-linearities. It consists in two steps. First, the Forward Propagation step considers the direct forms of the equation. Second, the Backward stage uses inversion forms of the functions appearing in the equation.

Let's consider the constraint ${ }^{1} \rho^{j}=$ $\sqrt{\left(x_{1}-x_{j}\right)^{2}+\left(y_{1}-y_{j}\right)^{2}}$ which was predefined in $\mathrm{Eq}$ (III.3) but without considering the noise and the bias. It is first decomposed into primitive constraints. A primitive constraint only involves an arithmetic operator or a usual function (sin, cos, etc.). For example, the decomposition into primitives constraints $\left(i_{1}, \cdots, i_{7}\right)$ will be shown in figure (III.3).

Please notice that the order of constraint is globally optimal here. After using the Forward-Backward propagation for each $\mathcal{H}$, we apply then the Waltz algorithm [14] with all the constraints each one providing consistent boxes with the considered measurement. Its principle is to repeat the propagation until the intervals do not contract any more.

Figure (III.4) illustrates the stage of the contraction of the predicted partner position 2 which is done in Robot $1\left({ }^{1} x_{2, k}\right)$ and the estimated position of Robot 2 which is done in Robot $2\left({ }^{2} x_{2, k}\right)$ (algorithm 2$)$.

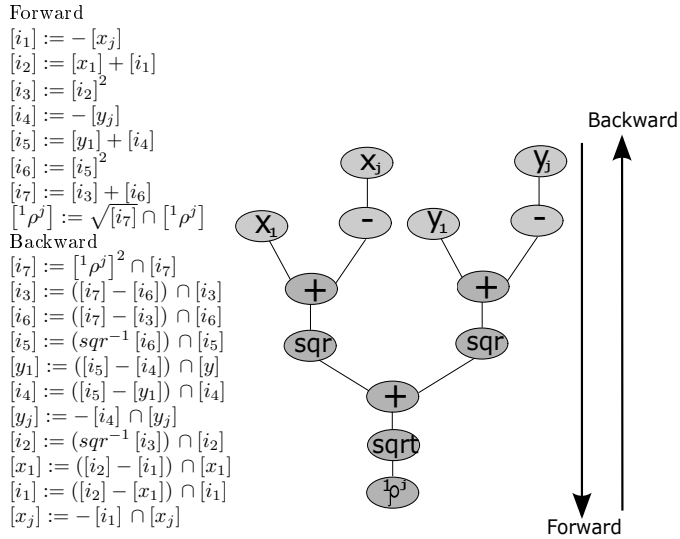

Figure III.3. Decomposition into primitives constraints associated to the measure of the distance between beacon $\mathrm{j}$ and robot 1

Algorithm 2 CP algorithm implemented in Robot 1

Initialization $(t=0)$ :

$\left[x_{1, k-1}\right]=\left[x_{1, \text { ref }}\right],\left[y_{1, k-1}\right]=\left[y_{1, \text { ref }}\right]$,

$\left[v_{1, k-1}\right]=\left[v_{1, \text { ref }}(0)\right],\left[\theta_{1, k-1}\right]=\left[\theta_{1, \text { ref }}(0)\right]$,

$\left[{ }^{1} b_{k-1}^{m}\right]=[0,5], \varepsilon$ (Tolerance of Contraction)

for $t=1$ to $T_{\max }$

State Prediction $\left[{ }^{1} X_{k}\right]$

$\left[x_{2, k-1}\right],\left[y_{2, k-1}\right],\left[v_{2, k-1}\right],\left[\theta_{2, k-1}\right]$

$\left[x_{1, k}\right]=\left[x_{1, k-1}\right]+T_{e}\left[v_{1, k-1}\right] \cos \left[\theta_{1, k-1}\right]$

$\left[y_{1, k}\right]=\left[y_{1, k-1}\right]+T_{e}\left[v_{1, k-1}\right] \sin \left[\theta_{1, k-1}\right]$

$\left[x_{2, k}\right]=\left[x_{2, k-1}\right]+T_{e}\left[v_{2, k-1}\right] \cos \left[\theta_{2, k-1}\right]$

$\left[y_{2, k}\right]=\left[y_{2, k-1}\right]+T_{e}\left[v_{2, k-1}\right] \sin \left[\theta_{2, k-1}\right]$

Every $\left[{ }^{1} b_{k}^{j}\right]$ remains the same $\left(\left[{ }^{1} b_{k}^{j}\right]=\left[{ }^{1} b_{k-1}^{j}\right]\right)$

State Estimation $\left[{ }^{1} X_{k}\right]$

while $\left(\max w i d t h\left(\left[{ }^{1} X_{k}\right]\right)<\varepsilon\right) d o$

for every measurement

$\left[{ }^{1} X_{k}\right]=$ Solve Forward-Backward CSP: $\{\mathcal{H}: F(\mathbf{x})=0\}$

end for

end while

Broadcast $\left(\left[{ }^{1} X_{k}\right],\left[v_{1, k}\right],\left[\theta_{1, k}\right]\right)$

if Received $\left(\left[{ }^{2} X_{k}\right],\left[v_{2, k}\right],\left[\theta_{2, k}\right]\right)$ then

$\left[{ }^{1} X_{k}\right]=\left[{ }^{1} X_{k}\right] \cap\left[{ }^{2} X_{k}\right]$ (Contract Positions and Biases)

endif

end for

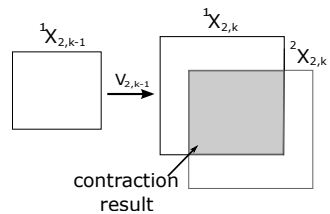

Figure III.4. Contraction of the partner position of Robot 2 estimated in Robot 1 and the received position of Robot 2 estimated in Robot 2

\section{Simulation RESUlTS}

In this section, we present the simulation results for the two scenarios: standalone and full cooperation localization. We aim to quantify the performance gain due to cooperation. The algorithm was tested by considering that the robots perform 
both circular trajectories with different radius $\left(r_{1}=70 \mathrm{~m}\right.$, $r_{2}=150 \mathrm{~m}$ ). Figure IV. 1 shows our scenarios. The right image presents the real position of each robot and the left one shows the simulation results and the inter-distance between the two robots. The simulation of scenarios is implemented using IBEX library for interval computation in real time.
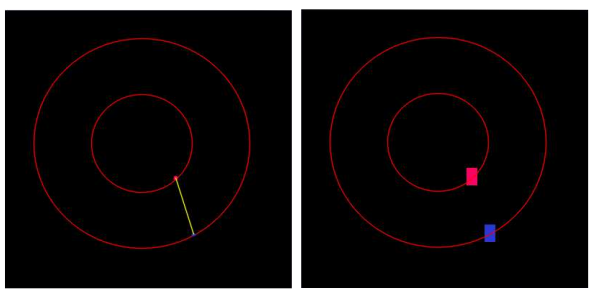

Figure IV.1. Robot 1 and Robot 2 performing circle trajectories

The number of beacons $m$ is equal to 40 and the beacons errors $b$ are considered as random positive values between 0 and 5 . The time of simulation $T_{\max }$ is 1000 seconds.

The values of variables noise are presented in table IV.1 :

\begin{tabular}{|c|c|}
\hline Variables & Noise bound of the measured variables \\
\hline \hline$\left[v_{k}\right]$ & {$\left[v_{k}-\left|\bar{\gamma}_{v}\right|, v_{k}+\left|\bar{\gamma}_{v}\right|\right] ; \bar{\gamma}_{v}=$ uniform noise $\times 0,28 \mathrm{~m} / \mathrm{s}$} \\
\hline$\left[\theta_{k}\right]$ & {$\left[\theta_{k}-\left|\bar{\gamma}_{\theta}\right|, \theta_{k}+\left|\bar{\gamma}_{\theta}\right|\right] ; \bar{\gamma}_{\theta}=$ uniform noise $\times 1{ }^{\circ}$} \\
\hline$\left[{ }^{i} \rho^{m}\right]$ & {$\left[{ }^{i} \rho^{m}-|\bar{\beta}|,{ }^{i} \rho^{m}+|\bar{\beta}|\right] ; \bar{\beta}=$ uniform noise $\times 1 \mathrm{~m}$} \\
\hline
\end{tabular}

THE DISTRIBUTION OF NOISE VARIABLES

Empirical cumulative distributed functions $F$ of the position errors are plotted in Figures (IV.2, IV.3) and the confidence domain size in Figures (IV.3, IV.4). The position and the confidence domain size of a robot are respectively the center and the volume of the estimated box. The method is guaranteed and the true values of all variables (position of robots, relative position of robots and biases) are always included in the estimated boxes. As shown in Figure (IV.2), when considering Robot $1,50 \%$ of position errors are less than $0.33 \mathrm{~m}$ and 0.65 $m$ when using FC method and standalone method respectively. $95 \%$ of these errors are less than $0.68 \mathrm{~m}$ and $1.13 \mathrm{~m}$ when using FC method and standalone method respectively. FC method improves significantly the standalone method in term of accuracy.

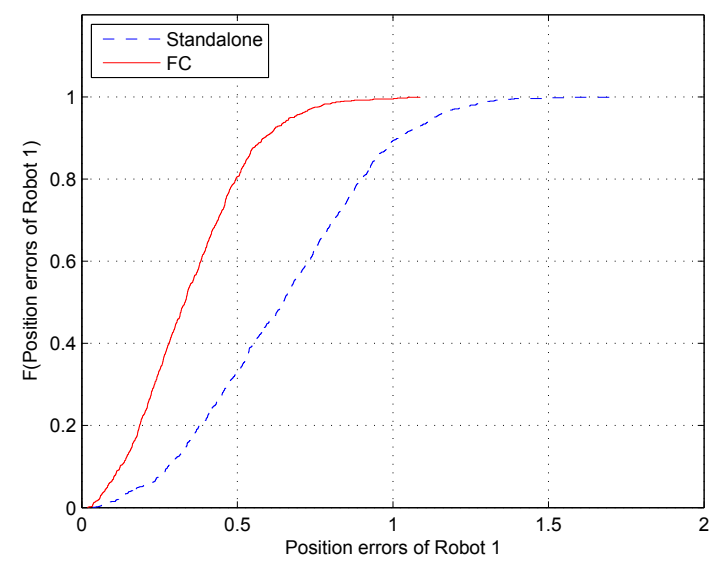

Figure IV.2. Position error of Robot 1 with standalone and full cooperation
As shown in Figure (IV.3), 95\% of position errors of Robot 2 which is estimated in the frame of Robot 1 are less than $0.67 \mathrm{~m}$ and $1.13 \mathrm{~m}$ when using FC and standalone method respectively. For some position errors, the center of box when using the standalone method is nearest to the solution than the center of estimated box with the cooperative method. This is due to the contraction step which can reduce the size of the box solution and modify the box. The representation above is not significant because we take the center of the box as the estimate, it is just an illustration example of position errors.

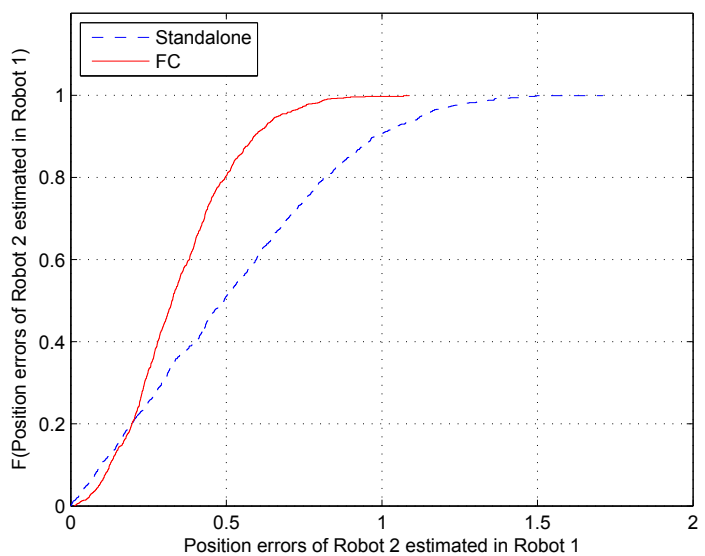

Figure IV.3. Position error of Robot 2 with standalone and full cooperation

As shown in Figure IV.4, 95\% of confidence domain size of Robot 1 is less than $7.84 \mathrm{~m}^{2}$ and $18.43 \mathrm{~m}^{2}$ when using the FC method and the standalone one respectively.

In IV.5, $95 \%$ of confidence domain size of Robot 2 , which is estimated in Robot 1 is less than $7.55 \mathrm{~m}^{2}$ and $18.13 \mathrm{~m}^{2}$ when using the FC method and the standalone one respectively. We can conclude that the FC method gives more precise confidence domain size of Robot 1 and of Robot 2 which is estimated in Robot 1.

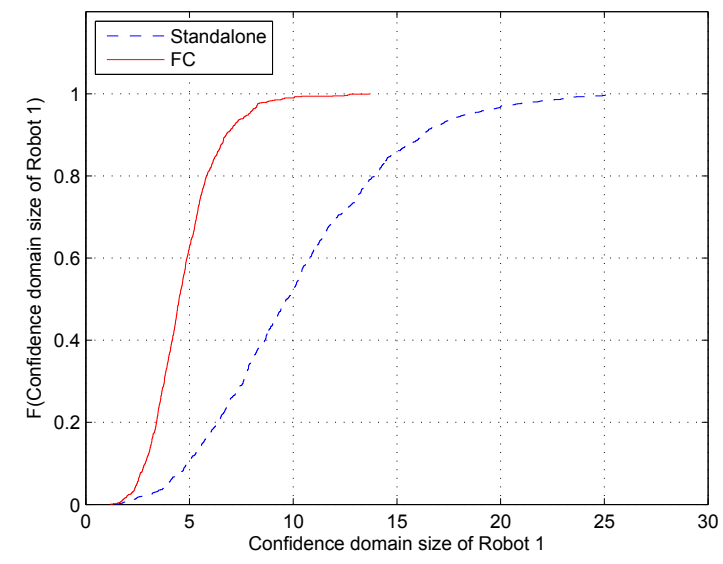

Figure IV.4. Confidence domain size of Robot 1 with standalone and full cooperation 


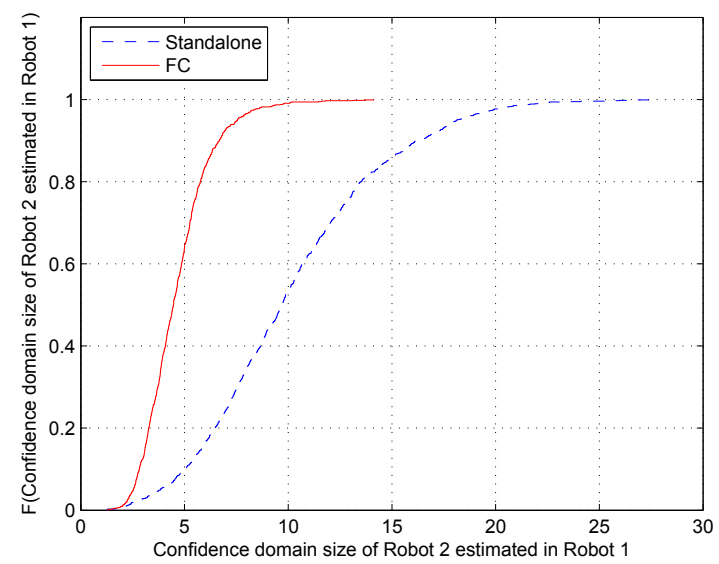

Figure IV.5. Confidence domain size of Robot 2 estimated in Robot 1 frame

The confidence size of inter-distance is also estimated using the step of State Estimation in the algorithm 2, by considering the state vector ${ }^{1} X_{k}$ as follows :

$$
\begin{gathered}
{ }^{1} X_{k}=\left[\begin{array}{lllll}
x_{1, k} & y_{1, k} & \triangle_{x, k} & \triangle_{y, k} & { }^{1} b_{k}^{m}
\end{array}\right]^{T} \\
\triangle_{x, k}=x_{1, k}-x_{2, k} \text { and } \triangle_{y, k}=y_{1, k}-y_{2, k}
\end{gathered}
$$

In figure IV.6, 95\% of confidence size of the inter-distance is less than $27.71 \mathrm{~m}$ and $192.8 \mathrm{~m}$ when using the FC method and the standalone one respectively.

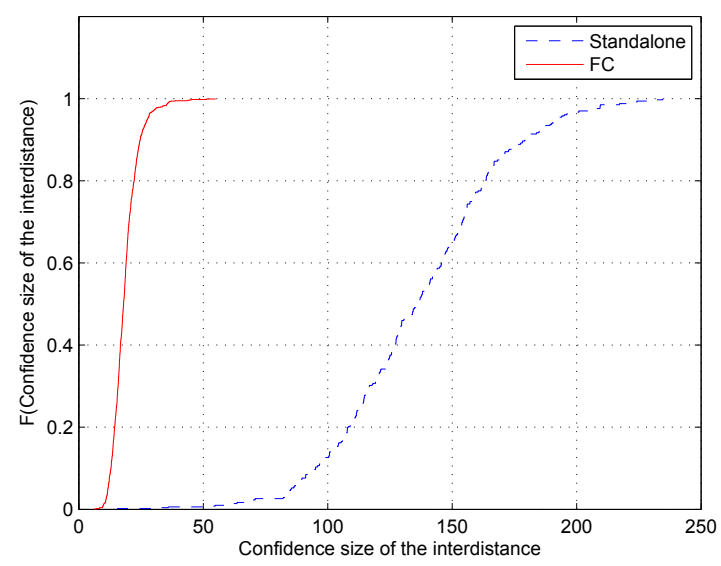

Figure IV.6. Confidence size of the interdistance

\section{CONCLUSION}

This paper has presented a Constraint Propagation approach to solve the cooperative localization problem in a team formed by two robots. By using a $\mathrm{CP}$ technique, the solution is guaranteed because it ensures the inclusion of the true value within the estimated bounds at each step.

The standalone and full cooperation methods were simulated and the results show the effectiveness of the proposed method for the cooperative localization in terms of accuracy and precision. As shown by the simulations, the confidence domain size of each robot and the confidence size of the inter-distance are significantly improved by the FC method compared to the standalone method. So, we can conclude that each robot is able to ameliorate its own absolute position and the relative position by information exchange, which is important in cooperative applications.

In our future work, we will extend this approach for heterogeneous systems particularly we will consider an aerial robot and a ground robot.

\section{ACKNOWLEDGMENT}

This work was carried out and funded in the framework of the Labex MS2T. It was supported by the French Government, through the "Investments for the future" program, managed by the National Agency for Research (Reference ANR-11-IDEX0004-02).

\section{REFERENCES}

[1] R. Madhavan, K. Fregene, and L. Parker, "Distributed heterogeneous outdoor multi-robot localization," IEEE International Conference on Robotics \& Automation, Washington DC, USA, pp.374-381, May 2002.

[2] H. Li, F. Nashashibi, and G. Toulminet, "Localization for intelligent vehicle by fusing mono-camera, low-cost GPS and map data," 13th International IEEE Annual Conference on Intelligent Transportation Systems, Madeira Island, Portugal, pp.1658-1662, September 2010.

[3] J. Laneurit, C. Blanc, R. Chapuis, and L. Trassoudaine, "Multisensorial data fusion for global vehicle and obstacles absolute positioning," Intelligent Vehicles Symposium, Aubière, France, pp.138-143, June 2003.

[4] N. Karam, R. Chapuis, R. Aufrere, and F. Chausse, "Localization of a Group of Communication Vehicles by State Exchange," IEEE/RSJ International Conference on Intelligent Robots and Systems, Beijing, China, pp.519-524, October 2006.

[5] L.Ronghua, T. Yasuda, "Cooperative Global Localization in Multi-robot System," INTECH, pp.131-148, Shanghai, China, January 2011.

[6] A. Ahmand, G. Diego, P. Lima, and W. Burgard, "Cooperative Robot Localization and Target Tracking based on Least Squares Minimization," IEEE International Conference on Robotics and Automation, Karlsruhe, Germany, pp.5969-5701, May 2013.

[7] R. Aufrere, N. Karam, F. Chausse, and R. Chapuis, "A state exchange approach in real conditions for multi-robot cooperative localization," IEEE/RSJ International Conference on Intelligent Robots and Systems, Taipei, Taiwan, pp.4346-4351, October 2010.

[8] D. Meizel, O. Leveque, L. Jaulin, and E. Walter, "Initial localization by set inversion," IEEE Transactions on Robotics and Automation, France, vol. 18, No.6, pp.966-971, December 2002.

[9] A. Gning, and P. Bonnifait, "Constraint propagation techniques on intervals for a guaranteed localization using redundant data," Automatica, France, vol. 42, pp.1167-1175, July 2006.

[10] V. Drevelle, L. Jaulin, and B. Zerr, "Guaranteed Characterization of the Explored Space of a Mobile Robot by using Subpavings," 9th IFAC Symposium on Nonlinear Control Systems, Toulouse, France, pp.44-49, September 2013.

[11] J. Kyoung-Hwan, and L. Jihong, "Cooperative Localization of Multiple Robots with Constraint Propagation Technique," IEEE/RSJ International Conference on Intelligent Robots and Systems, Nice, France, pp.34773482, September 2008.

[12] L. Jaulin, M. Kieffer, O. Didrit, and E. Walter, "Applied interval analysis with examples in parameter and state estimation, robust control and robotics," Springer, pp.65-100, 2001.

[13] Benhamou, F., Goualard, F., Granvilliers, L., and Puget, J. F. "Revising hull and box consistency," Proceedings of the intervational conference on logic programming, Las Cruces, NM, pp. 230-244, 1999.

[14] Waltz, D., "Generating semantic descriptions from drawings of scenes with shadows," The psychology of computer vision, New York, NY, pp. 19-91, 1975. 